\title{
P-48
}

\section{Chemical Constituents and Bioactivity Studies of Ardisia elliptica}

\author{
Nurul Zawani Alias* and Nur Kamisah Mohd Ishak \\ Faculty of Applied Sciences, Universiti Teknologi MARA (Perlis), 02600 Arau, Perlis, Malaysia; \\ E-mail: zawani299@perlis.uitm.edu.my
}

Ardisia elliptica is a medicinal plant traditionally used for alleviating chest pains, treatment of fever, diarrhea, liver poisoning and for parturition complications. The objectives of this study were to investigate the chemical constituents in leaves and fruits extracts of $A$. elliptica and to determine the biological activities of these extracts. The leaves and fruits of $A$. elliptica were soaked with methanol and the extracts were analysed using GCMS. Both extracts were then tested for antibacterial activity using agar well diffusion method against Staphylococcus aureus (gram positive) and Eschterichia coli (gram negative). After incubation for 24 hours, the inhibition zones of the extracts were compared with gentamicin and ampicilin. Antioxidant activities were determined using DPPH and analysed by Spectronic 20 with wavelength of 517 nm. From GCMS data, it was found that both extracts contained similar major compound which were 5-hydroxymethyl-2-furancarboxaldehyde with percentage area of $58.42 \%$, and $45.13 \%$; 2,4-di-tert-butylphenol with $3.04 \%$ and $20.87 \%$ and clindamycin with $8.77 \%$ and $5.79 \%$ for fruits and leaves extracts respectively. Clindamycin is a known antibiotic therefore both extracts showed positive result for antibacterial tests. Antioxidant assay also showed that both fruits and leaves extracts decolorized the DPPH.

Keywords: Ardisia elliptica, Clindamycin, DPPH. 